\title{
On Quantized Consensus by Means of Gossip Algorithm - Part II: Convergence Time
}

\author{
Javad Lavaei and Richard M. Murray
}

\begin{abstract}
This paper deals with the distributed averaging problem over a connected network of agents, subject to a quantization constraint. It is assumed that at each time update, only a pair of agents can update their own numbers in terms of the quantized data being exchanged. The agents are also required to communicate with one another in a stochastic fashion. In the first part of the paper, it was shown that the quantized consensus is reached by means of a stochastic gossip algorithm proposed in a recent paper, for any arbitrary quantization. The current part of the paper considers the expected value of the time at which the quantized consensus is reached. This quantity (corresponding to the worst case) is lower and upper bounded in terms of the topology of the graph, for uniform quantization. In particular, it is shown that the upper bound is related to the principal minors of the weighted Laplacian matrix. A convex optimization is also proposed to determine the set of probabilities (used to pick a pair of agents) which leads to the fast convergence of the gossip algorithm.
\end{abstract}

\section{INTRODUCTION}

During the past few decades, there has been a particular interest in the area of distributed computations, which aims to compute some quantity over a network of processors in a decentralized fashion [1], [2]. The distributed averaging problem, as a particular case, is concerned with computing the average of numbers owned by the agents of a group [3], [4]. This problem has been investigated through the notion of consensus in several papers, motivated by different applications [5], [6], [7], [8], [9], [10]. For instance, the synchronization of coupled oscillators, arising in biophysics, neurobiology, and systems biology, is studied in [5] and [6] to explore how to reach a consensus on the oscillation frequencies of all agents. Moreover, the problem of aligning the heading angles of a group of mobile agents (e.g. a flock of birds) is treated in [11]. Given a sensor network comprising a set of sensors measuring the same quantity in a noisy environment, the problem of consensus on state estimates is discussed in [12]. The consensus problem for networks of dynamic agents with fixed and switching topologies is tackled in [3], where it is shown that the convergence rate is related to the algebraic connectivity of the network. The work [13] elaborates on the relationship between the amount of information exchanged by the agents and the rate of convergence to the consensus. A more complete survey on this topic is given in the recent paper [4].

Consider the distributed average consensus in which the values owned by the agents are to be averaged in a distributed

This work has been supported by AFOSR and Air Force MURI.

The authors are with the Department of Control and Dynamical Systems, California Institute of Technology, Pasadena, USA (emails: lavaei@cds.caltech.edu; murray@cds.caltech.edu). fashion. Since it may turn out in some applications that all agents cannot update their numbers synchronously, the gossip algorithm has been widely exploited by researchers to handle the averaging problem asynchronously [14], [15]. This type of algorithm selects a pair of agents at each time instance, and updates their values based on some averaging policy. The consensus problem in the context of gossip algorithm has been thoroughly investigated in the literature [16], [17], [18], [19]. For instance, the work [16] studies the convergence of a general randomized gossip algorithm, and derives conditions under which the algorithm converges. That paper also shows that the averaging time of a gossip algorithm depends on the second largest eigenvalue of a doubly stochastic matrix characterizing the algorithm.

In light of communication constraints, the data being exchanged between each pair of agents is normally quantized. This has given rise to the emergence of quantized gossip algorithms. The notion of quantized consensus is defined in [18] for the case when quantized values (integers) are to be averaged over a connected network with digital communication channels. This paper shows that the quantized gossip algorithm leads to reaching the quantized consensus. This result is extended in [19] to the case when the quantization is uniform, and the initial numbers owned by the agents are reals (as opposed to being integers). The paper [19] shows that the quantized gossip algorithm works for a particular choice of the updating parameter, although it conjunctures that this result is true for a wide range of updating parameters. A related paper on quantized consensus gives a synchronous algorithm in order to reach a consensus with arbitrary precision, at the cost of not preserving the average of the initial numbers [20].

In this paper, a weighted connected graph is considered together with a set of scalars sitting on its vertices. The weight of each edge represents the probability of establishing a communication between its corresponding vertices through the updating procedure. It was shown in Part I of this work that the quantized consensus is reached under the stochastic gossip algorithm proposed in [19], for a wide range of updating parameters and any arbitrary quantizer including uniform and logarithmic ones [21]. The current part of the paper is concerned with the convergence time of the gossip algorithm. More precisely, consider the expected value of the time at which the consensus is reached, and take its maximum over all possible initial states belonging to a given hypercube. Lower and upper bounds on the this quantity are provided for a uniform quantizer, which turn out to be related to the Laplacian of the weighted graph. The upper bound is 
then minimized in order to obtain the best weights resulting in a small convergence time. To do so, a convex optimization problem is proposed, which can be solved by a semidefinite program.

This paper is organized as follows. Some preliminaries are presented in Section II, and the problem is formulated accordingly. The main results on the convergence time are provided in Section III. The results are then illustrated in Section IV with a numerical example. Later on, some concluding remarks are drawn in Section V. A number of proofs are finally given in an appendix.

\section{PROBLEM FORMULATION AND PRELIMINARIES}

Consider a connected weighted graph $\mathcal{G}=(\mathcal{V}, \mathcal{E}, \mathcal{P})$, where:

- $\mathcal{V}:=\left\{v_{1}, v_{2}, \ldots, v_{\nu}\right\}$ is the set of vertices of $\mathcal{G}$;

- $\mathcal{E}$ is the set of undirected edges of $\mathcal{G}$;

- $\mathcal{P}:=\left\{p_{i j}\right\}_{i, j}$ is the set of weights assigned to the edges of $\mathcal{G}$.

Assume that:

- The quantity $\sum p_{i j}$ is equal to 1 , where the sum is taken over all numbers $i, j \in \nu:=\{1,2, \ldots, \nu\}$ such that $i \leq j$.

- The number $p_{i j}(i, j \in \boldsymbol{\nu})$ is equal to zero if $(i, j) \notin \mathcal{E}$; otherwise, it is strictly positive. In particular, $p_{11}, p_{22}, \ldots, p_{\nu \nu}$ are all equal to zero.

The scalar $p_{i j}$ associated with the edge $(i, j)$ represents the probability of choosing the edge $(i, j)$ when an edge of $\mathcal{G}$ is to be picked at random. Suppose that a real number $x_{i}$ has been assigned to the vertex $v_{i}$, for all $i \in \nu$. Let $q(x)$ : $\mathbf{R} \rightarrow \mathbf{R}$ be a general quantization operator characterized as follows:

$$
q(x)=\left\{\begin{array}{ll}
L_{i} & \text { if } \quad x \in\left[L_{i}, \bar{L}_{i}\right] \\
L_{i+1} & \text { if } \quad x \in\left(\bar{L}_{i}, L_{i+1}\right]
\end{array} \quad \forall i \in \mathbf{Z}\right.
$$

where $\left\{L_{i}\right\}_{-\infty}^{\infty}$ is a monotonically increasing sequence of integers representing the quantization levels, and:

$$
\bar{L}_{i}:=\frac{L_{i}+L_{i+1}}{2}, \quad \forall i \in \mathbf{Z}
$$

Note that $\mathbf{Z}$ denotes the set of integers. In what follows, a quantized stochastic gossip algorithm is presented [19].

\section{Stochastic Gossip (SG) Algorithm:}

Step 1: Given a positive real $\varepsilon$, set $k=0$. Define $x_{i}[0]:=x_{i}$, for any $i \in \nu$.

Step 2: Pick an edge of $\mathcal{G}$ at random.

Step 3: Suppose that the ending vertices of the edge selected in Step 2 possess the values $x_{i}[k]$ and $x_{j}[k]$. Perform the following updates:

$$
\begin{aligned}
& x_{i}[k+1]=x_{i}[k]+\varepsilon \times\left(q\left(x_{j}[k]\right)-q\left(x_{i}[k]\right)\right), \\
& x_{j}[k+1]=x_{j}[k]+\varepsilon \times\left(q\left(x_{i}[k]\right)-q\left(x_{j}[k]\right)\right), \\
& x_{q}[k+1]=x_{q}[k], \quad \forall q \in \boldsymbol{\nu} \backslash\{i, j\}
\end{aligned}
$$

Step 4: Increase $k$ by 1 and jump to Step 2 .

Let the short-hand notation:

$$
\mathbf{x}[k]=\left[\begin{array}{llll}
x_{1}[k] & x_{2}[k] & \cdots & x_{\nu}[k]
\end{array}\right], \quad k \in \mathbf{Z}
$$

be used hereafter. Observe that the SG algorithm is stochastic in the sense that an edge must be chosen at random at each time update. The deterministic version of this algorithm, referred to as the deterministic gossip (DG) algorithm, can be obtained by replacing its step 2 with the following:

Step 2: Pick an edge of $\mathcal{G}$ arbitrarily (at the discretion of the user).

Throughout this paper, the symbol $\mathcal{G}(\mathcal{V}, \mathcal{E}, \mathcal{P})$ refers to the weighted graph $\mathcal{G}$, whereas the symbol $\mathcal{G}(\mathcal{V}, \mathcal{E})$ refers to the graph $\mathcal{G}$ with the weights on its edges removed.

Definition 1: Given a quantization-based protocol $\mathcal{C}$ acting on $\mathcal{G}(\mathcal{V}, \mathcal{E})$, denote with $\mathbf{x}[k], k \in \mathbf{N} \cup\{0\}$, the vector of values on the vertices of $\mathcal{G}$ at time $k$, obtained using this protocol. It is said that the quantized consensus is reached for the graph $\mathcal{G}$ under the protocol $\mathcal{C}$ if for every arbitrary initial state $\mathbf{x}[0] \in \mathbf{R}^{\nu}$, there exist a natural number $k_{0}$ and an integer $\mu$ such that either of the following sets of relations holds:

$$
\left\{\begin{array}{c}
\sum_{i=1}^{\nu} x_{i}[k]=\sum_{i=1}^{\nu} x_{i}[0] \\
x_{j}[k] \in\left[L_{\mu}, L_{\mu+1}\right]
\end{array} \quad \forall k \geq k_{0}, \forall j \in \nu\right.
$$

or:

$$
\left\{\begin{array}{c}
\sum_{i=1}^{\nu} x_{i}[k]=\sum_{i=1}^{\nu} x_{i}[0] \\
x_{j}[k] \in\left(\bar{L}_{\mu}, \bar{L}_{\mu+1}\right]
\end{array} \quad \forall k \geq k_{0}, \forall j \in \nu\right.
$$

In line with the above definition, if the protocol $\mathcal{C}$ is stochastic, one would say that the quantized consensus is reached almost surely if there exists a number $k_{0} \in \mathbf{N}$, with probability 1, for which either of the relations (5) or (6) holds. For simplicity, the short name consensus is used hereafter in order to refer to quantized consensus. A few definitions and notations will be introduced in the sequel.

Definition 2: Define $\mathcal{S}$ to be the set of all $\nu$-tuple $\left(\alpha_{1}, \alpha_{2}, \ldots, \alpha_{\nu}\right)$ such that $\alpha_{i} \in\left[x_{\min }, x_{\max }\right]$ and, in addition, $\alpha_{i}-x_{i}$ is an integer multiple of $\varepsilon$, for all $i \in \nu$, where:

$$
x_{\max }:=\max _{i \in \boldsymbol{\nu}}\left\lceil x_{i}\right\rceil, \quad x_{\min }:=\min _{i \in \boldsymbol{\nu}}\left\lfloor x_{i}\right\rfloor
$$

(The notations $\lceil\cdot\rceil$ and $\lfloor\cdot\rfloor$ stand for the ceiling and floor operators, respectively).

Definition 3: Let $\eta_{1}$ and $\eta_{2}$ be:

$$
\begin{array}{lll}
\eta_{1}=\max _{i \in \mathbf{Z}} \bar{L}_{i} & \text { s.t. } & \bar{L}_{i} \leq x_{\mathrm{ave}}, \\
\eta_{2}=\min _{j \in \mathbf{Z}} \bar{L}_{j} & \text { s.t. } & \bar{L}_{j} \geq x_{\mathrm{ave}}
\end{array}
$$

where $x_{\mathrm{ave}}:=\frac{x_{1}+x_{2}+\cdots+x_{\nu}}{\nu}$.

Definition 4: Define:

$$
\mathcal{S}_{o}:=\left\{\left(\alpha_{1}, \alpha_{2}, \ldots, \alpha_{\nu}\right) \in \mathcal{S} \mid \alpha_{i} \in\left(\eta_{1}, \eta_{2}\right], \quad \forall i \in \boldsymbol{\nu}\right\}
$$

Furthermore, let $\mathcal{S}_{o}\left(\bar{L}_{i}\right), i \in \mathbf{Z}$, be defined as the set of all $\nu$-tuple $\left(\alpha_{1}, \alpha_{2}, \ldots, \alpha_{\nu}\right) \in \mathcal{S}$ such that:

$$
\alpha_{j} \in\left(\bar{L}_{i}-\varepsilon\left(L_{i+1}-L_{i}\right), \bar{L}_{i}+\varepsilon\left(L_{i+1}-L_{i}\right)\right], \quad \forall j \in \boldsymbol{\nu}
$$


$\mathbf{Z}$ as:

Definition 5: Define the distance function $d_{\varepsilon}\left(\cdot, \mathcal{S}_{o}\right): \mathcal{S} \rightarrow$

$$
d_{\varepsilon}\left(\boldsymbol{\alpha}, \mathcal{S}_{o}\right):=\min _{\boldsymbol{\beta} \in \mathcal{S}_{o}} \frac{|\boldsymbol{\alpha}-\boldsymbol{\beta}|_{1}}{\varepsilon}, \quad \forall \boldsymbol{\alpha} \in \mathcal{S}
$$

where $|\cdot|_{1}$ denotes the $L_{1}$ norm. Define also the distance function $d_{\varepsilon}\left(\cdot, \mathcal{S}_{o}(\mu)\right)$ in the same vein.

The next result was proved in Part I of the paper [21].

Theorem 1: Given $\varepsilon \in(0,0.5]$, apply the SG algorithm to the graph $\mathcal{G}(\mathcal{V}, \mathcal{E}, \mathcal{P})$ with the initial state $\mathbf{x}[0]$. There exists a positive number $k_{1}$ for which one of the following cases takes place with probability 1 :

i) $\mathbf{x}[k]$ belongs to the set $\mathcal{S}_{o}$, for every $k \geq k_{1}$.

ii) $\mathbf{x}[k]$ belongs to the set $\mathcal{S}_{o}\left(\eta_{1}\right)$, for every $k \geq k_{1}$.

iii) $\mathbf{x}[k]$ belongs to the set $\mathcal{S}_{o}\left(\eta_{2}\right)$, for every $k \geq k_{1}$.

It is noteworthy that the above theorem proves reaching the consensus and, besides, describes the steady-state behavior of the system. In order to study the convergence time of the SG algorithm, it is desired to find lower and upper bounds on the quantity $k_{1}$ introduced in Theorem 1 . Throughout the rest of this paper, assume that $q(x)$ is a uniform quantizer, i.e., it rounds each real number $x$ to its nearest integer (by convention, assume that $q(r+0.5)=r$, for all integers $r$ ).

\section{CONVERGENCE TIME}

Since the SG algorithm is stochastic, the quantity $k_{1}$ introduced in Theorem 1 is a random variable. Define $t_{c}(\varepsilon)$ to be equal to $\max \mathcal{E}\left\{k_{1}\right\}$, where the maximum is taken over all initial states $\mathbf{x}[0] \in\left[x_{\min }, x_{\max }\right]^{\nu}$ that belong to none of the steady-state sets $\mathcal{S}_{o}, \mathcal{S}_{o}\left(\eta_{1}\right)$ and $\mathcal{S}_{o}\left(\eta_{2}\right)$ (note that $\mathcal{E}\{\cdot\}$ denotes the expectation operator). The term $t_{c}(\varepsilon)$ indeed quantifies the expected value of the convergence time in the worst case. This section aims to characterize $t_{c}(\varepsilon)$ in terms of $x_{\min }, x_{\max }, \varepsilon$, and the topology of the graph (together with the probabilities assigned to its edges).

Definition 6: Given an integer $r$, apply the SG (or DS) algorithm to the graph $\mathcal{G}(\mathcal{V}, \mathcal{E}, \mathcal{P})$ with the initial state $\mathbf{x}[0]$. The action of choosing an edge at time $k(k \in \mathbf{N})$ in step 2 of the SG (or DS) algorithm is said to be a positive action with respect to (w.r.t.) $d_{\varepsilon}\left(\cdot, \mathcal{S}_{o}(r+0.5)\right)$ if the inequality:

$$
d_{\varepsilon}\left(\mathbf{x}[k], \mathcal{S}_{o}(r+0.5)\right)<d_{\varepsilon}\left(\mathbf{x}[k-1], \mathcal{S}_{o}(r+0.5)\right)
$$

holds; otherwise, it is called a trivial action, meaning the following (see Lemma 2 in [21]):

$$
d_{\varepsilon}\left(\mathbf{x}[k], \mathcal{S}_{o}(r+0.5)\right)=d_{\varepsilon}\left(\mathbf{x}[k-1], \mathcal{S}_{o}(r+0.5)\right)
$$

Remark 1: Regarding Definition 6, one can observe that there is a reduction in the Lyapunov function $d_{\varepsilon}\left(\cdot, \mathcal{S}_{o}(r+\right.$ $0.5)$ ) by at least 1 during each positive action. Moreover, having assumed that the vertices $v_{i}$ and $v_{j}$ are chosen at time $k$, where $x_{i}[k-1]>x_{j}[k-1]$, it is straightforward to show that a positive action occurs at this time if and only if either of the following sets of relations holds:

- $x_{i}[k-1]>r+0.5+\varepsilon$ and $x_{j}[k-1] \leq r+0.5$; or

- $x_{i}[k-1]>r+0.5$ and $x_{j}[k-1] \leq r+0.5-\varepsilon$.

Definition 7: Given $\boldsymbol{\alpha} \in \mathbf{R}^{\nu}$ and $r \in \mathbf{Z}$, define $T_{r, \varepsilon}(\boldsymbol{\alpha})$ to be the time at which the first positive action is taken w.r.t. $d_{\varepsilon}\left(\cdot, \mathcal{S}_{o}(r+0.5)\right)$, provided the $\mathrm{SG}$ algorithm is applied to the graph $\mathcal{G}(\mathcal{V}, \mathcal{E}, \mathcal{P})$ with the initial state $\boldsymbol{\alpha}$. Notice that since the SG algorithm is stochastic, $T_{r, \varepsilon}(\boldsymbol{\alpha})$ is a random variable.

Definition 8: Given $\boldsymbol{\alpha} \in \mathbf{R}^{\nu}$, let $\mathcal{H}$ denote an infinite sequence whose elements all belong to $\mathcal{E}$ (i.e. $\mathcal{H}$ is an infinite sequence of edges). Define $T_{r, \varepsilon}(\boldsymbol{\alpha} \mid \mathcal{H})$ to be equal to the time when the first positive action is taken w.r.t. $d_{\varepsilon}\left(\cdot, \mathcal{S}_{o}(r+0.5)\right)$, provided that the DG algorithm is applied to the graph $\mathcal{G}(\mathcal{V}, \mathcal{E})$ with the initial state $\boldsymbol{\alpha}$, where the edge selected at time $k$ in step 2 of this algorithm is indeed the $k^{\text {th }}$ element of $\mathcal{H}$, for all $k \in \mathbf{N}$.

Definition 9: For every integer $r$ and infinite sequence of edges $\mathcal{H}$, define:

$$
\zeta(\varepsilon, r, \mathcal{H}):=\max T_{r, \varepsilon}(\boldsymbol{\beta} \mid \mathcal{H})
$$

where the maximum is taken over all $\nu$-tuple $\boldsymbol{\beta}:=$ $\left[\begin{array}{lll}\beta_{1} & \cdots & \beta_{\nu}\end{array}\right] \in\left[x_{\min }, x_{\max }\right]^{\nu}$ with the following properties:

- $\boldsymbol{\beta} \notin \mathcal{S}_{o}(r+0.5)$;

- There exist $i, j \in \boldsymbol{\nu}$ such that:

$-\beta_{i}>r+0.5+\varepsilon$ and $\beta_{j} \leq r+0.5$; or

$-\beta_{i}>r+0.5$ and $\beta_{j} \leq r+0.5-\varepsilon$.

Theorem 2: Given an integer $r$ and an infinite sequence of edges $\mathcal{H}$, there exists a vector $\boldsymbol{\alpha}:=\left[\begin{array}{lll}\alpha_{1} & \cdots & \alpha_{\nu}\end{array}\right]$ such that:

$$
\begin{aligned}
\zeta(\varepsilon, r, \mathcal{H}) & =T_{0,0.5}(\boldsymbol{\alpha} \mid \mathcal{H}), \\
\left\{\alpha_{1}, \alpha_{2}, \ldots, \alpha_{\nu}\right\} & =\{0.25,0.75, \ldots, 0.75,1.25\}
\end{aligned}
$$

where that the number 0.75 appears $\nu-2$ times in the set given above

Proof: The proof is based on a series of Lemmas provided in the appendix.

Theorem 2 states that the quantity $\zeta(\varepsilon, r, \mathcal{H})$, introduced in Definition 9 , is independent of $r$ and $\varepsilon$. Instead, it is continent upon only $\mathcal{H}$ and $\mathcal{G}$.

Define $\Phi$ as follows:

$$
\Phi:=\max \mathcal{E}\left\{T_{0,0.5}(\boldsymbol{\alpha})\right\}
$$

where the maximum is taken over all $\nu$-tuple $\boldsymbol{\alpha}=$ $\left[\begin{array}{lll}\alpha_{1} & \cdots & \alpha_{\nu}\end{array}\right]$ satisfying the relation $\left\{\alpha_{1}, \alpha_{2}, \ldots, \alpha_{\nu}\right\}=$ $\{0.25,0.75, \ldots .0 .75,1.25\}$ in which the value 0.75 appears $\nu-2$ times. The following theorem presents one of the main results of this section.

Theorem 3: Given a real $\varepsilon \in(0,0.5]$, the quantity $t_{c}(\varepsilon)$ can be lower and upper bounded as follows:

$$
\Phi \leq t_{c}(\varepsilon) \leq \frac{\nu\left(x_{\max }-x_{\min }+2\right) \Phi}{\varepsilon}
$$

Proof: It follows immediately from the definition of $\Phi$ that this quantity is a lower bound on $t_{c}(\varepsilon)$. To prove the other part of the inequality, assume with no loss of generality that:

$$
x_{\mathrm{ave}}-x_{\min } \geq x_{\max }-x_{\mathrm{ave}}
$$

Recall that the proof of Theorem 2 in Part I of the paper introduces two storage functions $V_{1}[k]$ and $V_{2}[k]$. It also suggests minimizing $V_{1}[k]$ until it reaches a constant level, 
and subsequently minimizing $V_{2}[k]$ (in other words, the edge being selected in step 2 of the algorithm at each update is to be chosen in such a way that the storage function is minimized). It follows from inequality (17) and the relation $\left|x_{\text {ave }}-\eta_{1}\right| \leq 1$ that:

$$
V_{1}[0] \leq \sum_{i=1}^{\nu} \frac{\left|x_{i}-\eta_{1}\right|}{\varepsilon} \leq \nu \frac{x_{\mathrm{ave}}-x_{\min }+1}{\varepsilon}
$$

At time $k=k_{0}$ where the minimum of $V_{1}[k]$ is reached, two possibilities can occur according to Lemma 3 in Part I of the paper. The first one is that $\mathbf{x}\left[k_{0}\right] \in \mathcal{S}_{o}\left(\eta_{1}\right)$, which implies that the consensus is reached, and there is no need to minimize $V_{2}[k]$ any longer. The second scenario is that the relation $x_{i}\left[k_{0}\right]>\eta_{1}$ holds for all $i \in \boldsymbol{\nu}$. Assume that the latter one is the case. It is easy to verify that:

$$
V_{2}\left[k_{0}\right] \leq \sum_{i=1}^{\nu} \frac{\left|x_{i}\left[k_{0}\right]-\eta_{2}\right|}{\varepsilon} \leq \nu \frac{x_{\max }-x_{\mathrm{ave}}+1}{\varepsilon}
$$

As a result:

$$
V_{1}[0]+V_{2}\left[k_{0}\right] \leq \frac{\nu\left(x_{\max }-x_{\min }+2\right)}{\varepsilon}
$$

On the other hand, the aforementioned discussion indicates that at most $V_{1}[0]+V_{2}\left[k_{0}\right]$ positive actions are required to reach the consensus. Moreover, it can be inferred from Theorem 2 that the expected value of the time between two consecutive positive actions is at most equal to $\Phi$ (until the consensus is reached). These facts along with inequality (20) complete the proof.

Theorem 3 implies that $t_{c}(\varepsilon)$ can be upper bounded by a term which is proportional to the inverse of $\varepsilon$. The question arises: how to compute $\Phi$ systematically? This is addressed in the sequel. The following definitions/notations will be convenient to proceed with the development of the paper.

- Let $P$ be the Laplacian of the weighted graph $\mathcal{G}$. In other words, $P$ is a $\nu \times \nu$ matrix whose $(i, j)$ off-diagonal entry, $i, j \in \boldsymbol{\nu}, i \neq j$, is equal to $-p_{i j}$ and its $(i, i)$ diagonal entry, $i \in \boldsymbol{\nu}$, is equal to $\sum_{k=1}^{\nu} p_{k i}$.

- For every $i \in \boldsymbol{\nu}$ and $M \in \mathbf{R}^{\nu \times \nu}$, define $M_{\sim i}$ to be the matrix obtained from $M$ by removing its $i^{\text {th }}$ row and $i^{\text {th }}$ column.

Theorem 4: The quantity $\Phi$ can be obtained as follows:

$$
\Phi=\max _{i \in \boldsymbol{\nu}}\left|\left(P_{\sim i}\right)^{-1} E\right|_{\infty}
$$

where $|\cdot|_{\infty}$ stands for the infinity norm, and $E \in \mathbf{R}^{\nu-1}$ is a vector of 1's.

Proof: For every $i, j \in \boldsymbol{\nu}, i \neq j$, let $\boldsymbol{\beta}_{i j}$ denote a $\nu$ dimensional vector whose elements are all equal to 0.75 , except for the $i^{\text {th }}$ and $j^{\text {th }}$ entries which are 0.25 and 1.25 , respectively. In addition, denote the quantity $\mathcal{E}\left\{T_{0,0.5}\left(\boldsymbol{\beta}_{i j}\right)\right\}$ with $\bar{t}_{c}(i, j)$ for simplicity. The goal is to contrive a recursive equation characterizing $\bar{t}_{c}(i, j)$. To this end, consider the graph $\mathcal{G}$ with the initial state $\boldsymbol{\beta}_{i j}$. The expected value of the time at which the first positive action is taken (under the SG algorithm) is, by definition, equal to $\bar{t}_{c}(i, j)$. To count this number in another way, run the algorithm one iteration.
Assume that the edge $e_{\mu}$ is chosen in this iteration, which leads to the following possibilities:

- $e_{\mu}$ is equal to the edge $(i, k)$, for some $k \in \nu \backslash\{j\}$ : In this case, due to the equality $\mathbf{x}[0]=\boldsymbol{\beta}_{i j}$, the vector $\mathbf{x}[1]$ is obtained as $\boldsymbol{\beta}_{k j}$. Hence, it is expected to take the first positive action after $\bar{t}_{c}(k, j)$ time updates (in addition to the first time update taken at the beginning).

- $e_{\mu}$ is equal to the edge $(i, j)$ : This means that a positive action is already taken at the first time update.

- $e_{\mu}$ is equal to the edge $(k, l)$, for some $k, l \in \nu \backslash\{i\}$ : In this case, it is easy to show that $\mathbf{x}[1]=\mathbf{x}[0]=\boldsymbol{\beta}_{i j}$. This implies that it is expected to take the first positive action after $\bar{t}_{c}(i, j)$ time updates (other than the first one already taken).

The above reasoning yields the recursive equation:

$$
\begin{aligned}
\bar{t}_{c}(i, j) & =1+\sum_{k \in \boldsymbol{\nu} \backslash\{j\}} p_{i k} \bar{t}_{c}(k, j) \\
& +\left(1-\sum_{k=1}^{\nu} p_{i k}\right) \bar{t}_{c}(i, j), \quad \forall i \in \boldsymbol{\nu} \backslash\{j\}
\end{aligned}
$$

Stack the scalars $\bar{t}_{c}(1, j), \ldots, \bar{t}_{c}(j-1, j), \bar{t}_{c}(j+1, j)$, $\ldots, \bar{t}_{c}(\nu, j)$ in a column and denote the resulting vector with $\overline{\mathbf{t}}_{c}(j) \in \mathbf{R}^{\nu-1}$. Equation (22) can be re-arranged as:

$$
P_{\sim j} \overline{\mathbf{t}}_{c}(j)=E, \quad \forall j \in \boldsymbol{\nu}
$$

Therefore:

$\Phi=\max _{i, j \in \boldsymbol{\nu}, i \neq j} \bar{t}_{c}(i, j)=\max _{j \in \boldsymbol{\nu}}\left|\overline{\mathbf{t}}_{c}(j)\right|_{\infty}=\max _{j \in \boldsymbol{\nu}}\left|\left(P_{\sim j}\right)^{-1} E\right|_{\infty}$

This completes the proof.

The results of Theorems 3 and 4 can be combined to explicitly bound the quantity $t_{c}(\varepsilon)$ as follows:

$$
\max _{j \in \boldsymbol{\nu}}\left|\left(P_{\sim j}\right)^{-1} E\right|_{\infty} \leq t_{c}(\varepsilon)
$$

and

$$
t_{c}(\varepsilon) \leq \frac{\nu\left(x_{\max }-x_{\min }+2\right)}{\varepsilon} \max _{j \in \boldsymbol{\nu}}\left|\left(P_{\sim j}\right)^{-1} E\right|_{\infty}
$$

The next theorem directly relates the upper bound on $t_{c}(\varepsilon)$ to the spectral of the principal submatrices of the Laplacian.

Theorem 5: The scalar $t_{c}(\varepsilon)$ satisfies the following inequality:

$$
t_{c}(\varepsilon) \leq \frac{\nu \sqrt{\nu-1}\left(x_{\max }-x_{\min }+2\right)}{\varepsilon}\left(\max _{i \in \boldsymbol{\nu}} \frac{1}{\lambda_{\min }\left\{P_{\sim i}\right\}}\right)
$$

where $\lambda_{\min }(\cdot)$ represents the smallest eigenvalue of a matrix.

Proof: One can write:

$$
\begin{aligned}
\left|\left(P_{\sim j}\right)^{-1} E\right|_{\infty} & \leq\left|\left(P_{\sim j}\right)^{-1}\right|_{\infty}|E|_{\infty} \\
& =\left|\left(P_{\sim j}\right)^{-1}\right|_{\infty} \leq \sqrt{\nu-1}\left|\left(P_{\sim j}\right)^{-1}\right|_{2}
\end{aligned}
$$

where $|\cdot|_{2}$ stands for the 2-norm. Since the weighted Laplacian matrix $P$ is positive semi-definite (PSD), its principal submatrix $P_{\sim j}$ is PSD too. As a result, it can be deduced from the above inequality that:

$$
\left|\left(P_{\sim j}\right)^{-1} E\right|_{\infty} \leq \sqrt{\nu-1} \frac{1}{\lambda_{\min }\left\{P_{\sim j}\right\}}
$$


The proof is completed by combining inequalities (26) and (29).

Remark 2: Theorem 5 states that the convergence time $t_{c}(\varepsilon)$ is related to the $(\nu-1)^{\text {th }}$ order submatrices of the Laplacian of the graph (i.e. $P_{\sim i}, i \in \nu$ ), rather than the Laplacian itself. Let $\lambda_{2}(P)$ denote the second smallest eigenvalue of $P$. Since the graph $\mathcal{G}$ is connected, $\lambda_{2}(P)$ is strictly positive. Now, the interlacing theorem can be exploited to argue that:

$$
0<\lambda_{\min }\left\{P_{\sim j}\right\} \leq \lambda_{2}(P)
$$

This means that unlike the unquantized consensus whose convergence mainly depends on $\lambda_{2}(P)$, a more subtle dependency on $\lambda_{2}(P)$ is governed for the quantized case (in fact, $\Phi$ is not directly related to $\lambda_{2}(P)$ ).

Remark 3: The quantity $\Phi$ introduced in this section is an important parameter, which characterizes the expected value of the maximum number of iterations that must be followed until a positive action is taken. The derived lower and upper bounds on $t_{c}(\varepsilon)$ have been related to this quantity corresponding to the worst case. Since the graph system is stochastic, it is unlikely in practice that the system operates in the worst case, which implies that the actual convergence time of a random system may be much better than the bounds obtained here. Nevertheless, these bounds are instrumental in understating the worst-case behavior of the system and how the convergence time is related to the topology of the graph.

\section{A. Special graphs}

This subsection aims to obtain lower and upper bounds on the quantity $t_{c}(\varepsilon)$ for both complete and path graphs in the case when all edges have the same weight. In this regard, assume that each edge is associated with the same weight $p$.

Corollary 1: For a complete graph $\mathcal{G}$ with equally weighted edges, the following inequality holds:

$$
\frac{\nu(\nu-1)}{2} \leq t_{c}(\varepsilon) \leq \frac{\nu^{2}(\nu-1)\left(x_{\max }-x_{\min }+2\right)}{2 \varepsilon}
$$

Proof: The weight $p$ for this graph is equal to $\frac{2}{\nu(\nu-1)}$. Using this fact and by means of Theorems 3 and 4 , it is straightforward to show the validity of inequality (31). The details are omitted here.

Corollary 2: Let $\mathcal{G}$ be a path graph with equally weighted edges so that $v_{i}$ is connected to $v_{i+1}$, for all $i \in\{1,2, \ldots, \nu-$ 1\} (these are the only edges of the graph). The inequality given below holds:

$$
\frac{\nu(\nu-1)^{2}}{2} \leq t_{c}(\varepsilon) \leq \frac{\nu^{2}(\nu-1)^{2}\left(x_{\max }-x_{\min }+2\right)}{2 \varepsilon}
$$

Proof: Since the graph $\mathcal{G}$ has $\nu-1$ edges, the weight $p$ is equal to $\frac{1}{\nu-1}$. On the other hand, it is easy to show that $\Phi=\bar{t}_{c}(1, \nu)$. This leads to the following recursive equations (in light of (22)):

$$
\begin{aligned}
& p \bar{t}_{c}(1, \nu)-p \bar{t}_{c}(2, \nu)=1 \\
& -p \bar{t}_{c}(i-1, \nu)+2 p \bar{t}_{c}(i, \nu)-p \bar{t}_{c}(i+1, \nu)=1 \\
& -p \bar{t}_{c}(\nu-2, \nu)+2 p \bar{t}_{c}(\nu-1, \nu)=1
\end{aligned}
$$

where the argument $i$ in equation (33b) belongs to the set $\{2,3, \ldots, \nu-2\}$. Adding up these equalities results in the relation:

$$
p \bar{t}_{c}(\nu-1, \nu)=\nu-1
$$

The (backward) recursive equation (33b) can be solved using conventional techniques to conclude that there exist two constants $a$ and $b$ such that:

$$
p \bar{t}_{c}(i, \nu)=a+b i-\frac{i^{2}}{2}, \quad i=\nu-1, \nu-2, \ldots, 1
$$

One can employ the final conditions given by (33c) and (34) to arrive at:

$$
a=\frac{\nu^{2}-\nu}{2}, \quad b=\frac{1}{2}
$$

This implies that:

$$
\Phi=\bar{t}_{c}(1, \nu)=\frac{\nu^{2}-\nu}{2 p}=\frac{\nu(\nu-1)^{2}}{2}
$$

The proof follows immediately from the above equation and Theorem 3.

\section{B. Optimal edge weights}

In this subsection, it is desired to find out what probabilities the edges of $\mathcal{G}$ should possess so that the consensus is reached quickly. For this purpose, observe that the quantity $t_{c}(\varepsilon)$ has been related to the spectral of the submatrices of the Laplacian in (27). Having fixed $x_{\min }, x_{\max }$ and $\varepsilon$, the provided upper bound on $t_{c}(\varepsilon)$ is a multiple of the term:

$$
\max _{i \in \boldsymbol{\nu}} \frac{1}{\lambda_{\min }\left\{P_{\sim i}\right\}}
$$

Therefore, it is desired to minimize the function (38) over all possible (discrete) probability distributions captured by $P$ for the sake of finding a sub-optimal edge-selection probability distribution. This is accomplished in the sequel.

Problem 1: Minimize the scalar variable $-\mu$ subject to the constraints:

$$
\lambda_{\min }\left\{P_{\sim i}\right\} \geq \mu, \quad i=1,2, \ldots, \nu
$$

where $P$ is a matrix variable representing the Laplacian of the weighted graph $\mathcal{G}$. Denote the global minimizer of this optimization with $\left(\mu^{*}, P^{*}\right)$ (note that there are some implicit constraints stating that the weights on the edges are positive and sum up to 1 ).

Since the operator $\lambda_{\min }(\cdot)$ is concave w.r.t. its symmetric argument, it is easy to show that Problem 1 is convex. More precisely, the constraint $\lambda_{\min }\left\{P_{\sim i}\right\} \geq \mu$ can be expressed as $P_{\sim i} \succeq \mu I$, which is a semidefinite constraint. Hence, the solution $P^{*}$ can be found efficiently. On the other hand, one can verify that:

$$
\mu^{*}=\max _{P} \min _{i} \lambda_{\min }\left\{P_{\sim i}\right\}
$$

or equivalently:

$$
\frac{1}{\mu^{*}}=\min _{P} \max _{i} \frac{1}{\lambda_{\min }\left\{P_{\sim i}\right\}}
$$


This implies that the solution $P^{*}$ gives a sub-optimal edgeselection probability distribution (resulting in the fast convergence of the SG algorithm), because of minimizing the term given in (38).

\section{NUMERICAL EXAMPLE}

Consider the graph $\mathcal{G}$ drawn in Figure 1. The objective is to find out what probabilities should be assigned to the edges of $\mathcal{G}$ so that the consensus is reached quickly under the SG algorithm. To this end, let the convex optimization provided in Problem 1 be solved. This yields the following probability distribution:

$$
\begin{aligned}
& p_{12}=p_{15}=0.2087 \\
& p_{23}=p_{24}=p_{45}=p_{35}=0.1146 \\
& p_{34}=0.1241
\end{aligned}
$$

The quantity $\Phi$ corresponding to this set of edge-selection

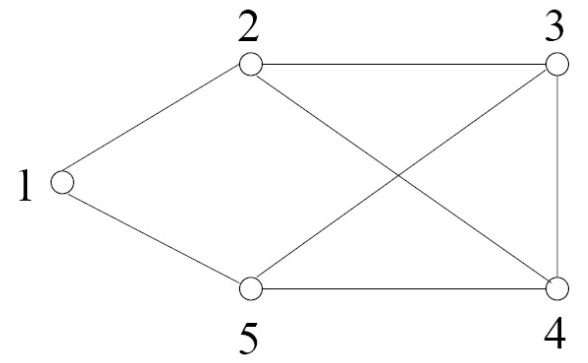

Fig. 1. The graph $\mathcal{G}$ rendered in the numerical example

probabilities turns out to be equal to 14.1770 . One can make a comparison with two heuristic methods for designing the probability set $\mathcal{P}$, which are spelled out below:

- The most naive approach is to assume that the edges of the graph are equally weighted. This leads to the probability $p=\frac{1}{7}$ on each edge. The associated quantity $\Phi$ is obtained as 17.5 .

- Another technique is to devise the probability distribution $\mathcal{P}$ in such a way that all vertices have the same probability of being chosen at each time update, i.e.:

$$
\begin{aligned}
p_{12}+p_{15} & =p_{21}+p_{23}+p_{24} \\
& =p_{32}+p_{34}+p_{35} \\
& =p_{42}+p_{43}+p_{45} \\
& =p_{51}+p_{53}+p_{54}
\end{aligned}
$$

Note that $p_{i j}=p_{j i}, \forall i, j \in \boldsymbol{\nu}$. The above set of equations has a unique symmetric solution (complying with the symmetry of the graph $\mathcal{G}$ ) as follows:

$$
\begin{aligned}
& p_{12}=p_{15}=0.2, \\
& p_{23}=p_{24}=p_{45}=p_{35}=0.1, \\
& p_{34}=0.2
\end{aligned}
$$

The corresponding $\Phi$ is equal to 15 .

Hence, the value of $\Phi$ corresponding to the sub-optimal solution is better from the ones obtained using these two rudimentary techniques.
An interesting fact about the edge selection can be seen in this example. Assume that the graph $\mathcal{G}$ does not have the edge $(1,5)$ (i.e. remove this edge). In this case, Problem 1 leads to the following solution:

$$
\begin{aligned}
& p_{12}=0.3781, p_{23}=p_{24}=0.1757, \\
& p_{34}=0, p_{53}=p_{54}=0.1352
\end{aligned}
$$

associated with $\Phi=23.1292$. Notice that $p_{34}=0$, which signifies that although a complete graph has the best convergence, if some edges do not exist (e.g. the edge $(1,5)$ ), it might be better to ignore some other edges too (e.g. the edge $(3,4))$. This is interesting as it reveals the fact that some communications are redundant.

To compare the value obtained for $\Phi$ with other possible values, consider the case when all edges have the same weight. This results in the equality $\Phi=36$. Therefore, there is a noticeable improvement in the value of $\Phi$ via the solution of Problem 1.

For the purpose of simulation, the following points have been randomly generated in the interval $[0,100]$ :

$$
\begin{aligned}
& x_{1}[0]=20.1185, x_{2}[0]=13.6221, x_{3}[0]=97.8356, \\
& x_{4}[0]=45.5033, x_{5}[0]=45.9224
\end{aligned}
$$

The stochastic gossip algorithm was run 1000 times and the average of the scalar $k_{1}$ was calculated accordingly (note that $k_{1}$ is a random variable). This value for the probability distribution (45) was obtained as 48.5710, while that for the identical probability distribution (equal edge weights) turned out to be 65.3580 . This demonstrates that one could significantly save in the convergence time if the solution of Problem 1 is employed, which also obviates the usage of the edge $(3,4)$.

\section{CONCLUSIONS}

This paper tackles the average consensus problem over a connected weighted graph subject to a quantization constraint. It is assumed that each pair of vertices can be chosen with a certain probability in order to update their numbers in term of the quantized data being exchanged. In the first part of the paper, it was shown that the quantized consensus is reached under the stochastic gossip algorithm given in a recent paper. This part of the paper deals with the time at which the consensus is reached. Lower and upper bounds on the expected value of this quantity in the worst case are provided, which depend on the principal minors of the Laplacian matrix of the weighted graph. These bounds are explicitly computed for equally weighted complete and path graphs. Finally, a convex optimization is provided to obtain a set of weights on the edges of the graph that results in the fast convergence of the gossip algorithm.

\section{REFERENCES}

[1] G. Tel, "Introduction to distributed algorithms," Cambridge University Press, 2000

[2] N. A. Lynch, "Distributed algorithms," Morgan Kaufmann Publishers, Inc., San Francisco, CA, 1996 
[3] R. Olfati-Saber and R. M. Murray, "Consensus problems in networks of agents with switching topology and time-delays," IEEE Transactions on Automatic Control, vol. 49, no. 9, pp. 1520-1533, 2004.

[4] R. Olfati-Saber, J. A. Fax and R. M. Murray, "Consensus and cooperation in networked multi-agent systems," Proceedings of the IEEE, vol. 95, no. 1, pp. 215-233, 2007.

[5] Y. Kuramoto, "Chemical oscillators, waves, and turbulance," SpringerVerlag, Berlin, 1984.

[6] S. H. Strogatz, "Exploring complex networks," Nature, vol. 410, pp. 268-276, 2001.

[7] D. P. Bertsekas and J. N. Tsitsiklis, "Parallel and distributed computation: Numerical methods," Belmont, MA: Athena Scientific, 1997.

[8] Y. Rabani, A. Sinclair and R. Wanka, "Local divergence of Markov chains and the analysis of iterative load-balancing schemes," in Proceedings of IEEE Conference on Foundations of Computer Science, 1998.

[9] A. V. Savkin, "Coordinated collective motion of groups of autonomous mobile robots: Analysis of Vicsekś model," IEEE Transactions on Automatic Control, vol. 49, no. 6, pp. 981982, 2004.

[10] R. Olfati-Saber, "Flocking for multi-agent dynamic systems: Algorithms and theory," IEEE Transactions on Automatic Control, vol. 51, no. 3, pp. 401420, 2006.

[11] A. Jadbabaie, J. Lin, and A. S. Morse, "Coordination of groups of mobile autonomous agents using nearest neighbor rules," IEEE Transactions on Automatic Control, vol. 48, no. 6, pp. 9881001, 2003.

[12] A. Speranzon, C. Fischione and K.H. Johansson, "Distributed and collaborative estimation over wireless sensor networks," in Proceedings of the 45th IEEE Conference on Decision and Control, 2006.

[13] R. Carli, F. Fagnani, A. Speranzon and S. Zampieri, "Communication constraints in the average consensus problem," Automatica, vol. 44, no. 3, pp. 671-684, 2008.

[14] J. Tsitsiklis, "Problems in decentralized decision making and computation," PhD thesis, Dept. of Electrical Engineering and Computer Science, M.I.T., Boston, MA, 1984.

[15] S. Boyd, A. Ghosh, B. Prabhakar and D. Shah , "Analysis and optimization of randomized gossip algorithms," in Proceedings of the 43rd IEEE Conference on Decision and Control, 2004.

[16] S. Boyd, A. Ghosh, B. Prabhakar and D. Shah, "Randomized gossip algorithms," IEEE Transactions on Information Theory, vol. 52, no. 6, pp. 2508-2530, 2006

[17] F. Benezit, A. G. Dimakis, P. Thiran and M. Vetterli, "Gossip along the way: Order-optimal consensus through randomized path averaging," in Proceedings of the Allerton Conference on Communication, Control, and Computing, 2007.

[18] A. Kashyap, T. Basara and R. Srikanta, "Quantized consensus," Automatica, vol. 43, no. 7, pp. 1192-1203, 2007.

[19] P. Frasca, R. Carli, F. Fagnani and S. Zampieri, "Average consensus by gossip algorithms with quantized communication," in Proceedings of the 47th IEEE Conference on Decision and Control, 2008.

[20] A. Censi and R. M. Murray, "A biologically inspired approach to real-valued average consensus over quantized channels with arbitrary deterministic accuracy," in Proceedings of the 2009 American Control Conference, 2009.

[21] J. Lavaei and R. M. Murray, "On quantized consensus by means of gossip algorithm - Part II: convergence time," in Proceedings of the 2009 American Control Conference, 2009.

\section{APPENDIX}

This appendix derives a number of results in order to prove Theorem 2.

Lemma 1: Given $r \in \mathbf{Z}$ and $\boldsymbol{\alpha}:=\left[\begin{array}{lll}\alpha_{1} & \cdots & \alpha_{\nu}\end{array}\right]^{T} \notin$ $\mathcal{S}_{o}(r+0.5)$, the following hold for every infinite sequence of edges $\mathcal{H}$ :

i) If $\alpha_{1}>r+0.5+\varepsilon$, then:

$$
T_{r, \varepsilon}(\boldsymbol{\alpha} \mid \mathcal{H}) \leq T_{r, \varepsilon}\left(\alpha_{1}-\varepsilon, \alpha_{2}, \ldots, \alpha_{\nu} \mid \mathcal{H}\right)
$$

ii) If $\alpha_{1} \leq r+0.5-\varepsilon$, then:

$$
T_{r, \varepsilon}(\boldsymbol{\alpha} \mid \mathcal{H}) \leq T_{r, \varepsilon}\left(\alpha_{1}+\varepsilon, \alpha_{2}, \ldots, \alpha_{\nu} \mid \mathcal{H}\right)
$$

Proof: Let case (i) be proved here, as the other case is analogous. Apply the DG algorithm to the graph $\mathcal{G}(\mathcal{V}, \mathcal{E})$ so that its step 2 selects edges from the sequence $\mathcal{H}$ in order. For the initial states $\left(\alpha_{1}, \alpha_{2}, \ldots, \alpha_{\nu}\right)$ and $\left(\alpha_{1}-\varepsilon, \alpha_{2}, \ldots, \alpha_{\nu}\right)$, denote the resulting numbers on the vertices of $\mathcal{G}$ at time $k$ with $\mathbf{w}[k]:=\left(w_{1}[k], w_{2}[k], \ldots, w_{\nu}[k]\right)$ and $\overline{\mathbf{w}}[k]:=$ $\left(\bar{w}_{1}[k], \bar{w}_{2}[k], \ldots, \bar{w}_{\nu}[k]\right)$, respectively, for all $k \in \mathbf{N} \cup\{0\}$. Furthermore, for notational simplicity, define:

$$
m:=T_{r, \varepsilon}\left(\alpha_{1}-\varepsilon, \alpha_{2}, \ldots, \alpha_{\nu} \mid \mathcal{H}\right)
$$

In order to proceed with the proof by contradiction, assume that there is no positive action w.r.t. $d_{\varepsilon}\left(\cdot, \mathcal{S}_{o}(r+0.5)\right)$ at time instants $1,2, \ldots, m$, by starting from the initial state $\boldsymbol{\alpha}$. In other words:

$$
d_{\varepsilon}\left(\mathbf{w}[0], \mathcal{S}_{o}(r+0.5)\right)=d_{\varepsilon}\left(\mathbf{w}[k], \mathcal{S}_{o}(r+0.5)\right)
$$

for any $k \in\{0,1,2, \ldots, m\}$. Now, one can draw a number of conclusions as follows:

i) $w_{1}[k]$ is always greater than or equal to $\bar{w}_{1}[k]$ for every $k$ satisfying the inequality $1 \leq k \leq m$.

ii) It is a consequence of property (i) that $w_{i}[k] \geq \bar{w}_{i}[k]$ for every $i \in \boldsymbol{\nu}$ and $k \in\{1, \ldots, m\}$.

iii) The relation $w_{i}[k]=\bar{w}_{i}[k]$ holds if $w_{i}[k] \leq r+0.5$ or $\bar{w}_{i}[k] \leq r+0.5$, for every $i \in \boldsymbol{\nu}$ and $k \in$ $\{0,1, \ldots, m-1\}$. This result can be easily proven by induction on $k$, taking property (ii) into account, and using the equality (50).

Assume that the $m^{\text {th }}$ element of $\mathcal{H}$ is the edge $(i, j)$. With no loss of generality, suppose that $\bar{w}_{i}[m-1] \geq \bar{w}_{j}[m-1]$. Since a positive action occurs at time $m$ w.r.t. $d_{\varepsilon}\left(\cdot, \mathcal{S}_{o}(r+\right.$ $0.5)$ ) by starting from the initial state $\overline{\mathbf{w}}[0]$, it follows from Remark 1 that either of the cases pointed out below occurs:

a) $\bar{w}_{i}[m-1]>r+0.5+\varepsilon$ and $\bar{w}_{j}[m-1] \leq r+0.5$; or b) $\bar{w}_{i}[m-1]>r+0.5$ and $\bar{w}_{j}[m-1] \leq r+0.5-\varepsilon$.

Assume that case (a) happens (the other case is similar). Properties (ii) and (iii) mentioned above yield that:

$$
\begin{aligned}
& w_{i}[m-1] \geq \bar{w}_{i}[m-1]>r+0.5+\varepsilon, \\
& w_{j}[m-1]=\bar{w}_{j}[m-1] \leq r+0.5
\end{aligned}
$$

Since the edge $(i, j)$ is chosen at time $m$ in step 2 of the DG algorithm, the above inequalities and Remark 1 signify that a positive action occurs at time $m$ for the graph $\mathcal{G}(\mathcal{V}, \mathcal{E})$ with the initial state $\boldsymbol{\alpha}=\mathbf{w}[0]$. In other words, $T_{r, \varepsilon}(\boldsymbol{\alpha} \mid \mathcal{H})$ must be equal to $m$, while it has been already assumed that this quantity is greater than $m$. This contradiction completes the proof.

Proposition 1: Given an integer $r$ and an infinite sequence of edges $\mathcal{H}$, there exist an integer $\kappa \in \boldsymbol{\nu}$ and a vector $\boldsymbol{\alpha}=$ $\left[\begin{array}{ccc}\alpha_{1} & \cdots & \alpha_{\nu}\end{array}\right]$ subject to:

$$
\begin{gathered}
\zeta(\varepsilon, r, \mathcal{H})=T_{r, \varepsilon}(\boldsymbol{\alpha} \mid \mathcal{H}), \\
r+0.5-\varepsilon<\alpha_{i} \leq r+0.5+\varepsilon, \quad \forall i \in \nu \backslash\{\kappa\}
\end{gathered}
$$

In addition, $\alpha_{\kappa}$ satisfies one of the following relations:

$$
r+0.5+\varepsilon<\alpha_{\kappa} \leq r+0.5+2 \varepsilon
$$

or:

$$
r+0.5-2 \varepsilon<\alpha_{\kappa} \leq r+0.5-\varepsilon
$$


Proof: The proof follows from Lemma 1. The details are omitted here for brevity. Note that the reason why inequality (52) is not satisfied for every $i$ in $\nu$ (i.e. there is a $\kappa$ for which this inequality does not hold) is that $\boldsymbol{\alpha}$ should not belong to $\mathcal{S}_{o}(r+0.5)$, in light of Definition 9 .

Lemma 2: Consider a vector $\boldsymbol{\alpha}=\left[\begin{array}{lll}\alpha_{1} & \cdots & \alpha_{\nu}\end{array}\right]$ and an integer $r \in \mathbf{Z}$ satisfying the relations:

$$
\begin{aligned}
& r+0.5+\varepsilon<\alpha_{1} \leq r+0.5+2 \varepsilon, \\
& r+0.5-\varepsilon<\alpha_{2} \leq r+0.5, \\
& r+0.5-\varepsilon<\alpha_{i} \leq r+0.5+\varepsilon, \quad \forall i \in \nu \backslash\{1,2\}
\end{aligned}
$$

For every infinite sequence of edges $\mathcal{H}$, the inequality given below holds:

$$
T_{r, \varepsilon}(\boldsymbol{\alpha} \mid \mathcal{H}) \leq T_{r, \varepsilon}\left(\alpha_{1}, \alpha_{2}+\varepsilon, \ldots, \alpha_{\nu} \mid \mathcal{H}\right)
$$

Proof: Apply the DG algorithm to the graph $\mathcal{G}(\mathcal{V}, \mathcal{E})$ and select edges in its step 2 from the sequence $\mathcal{H}$ successively. For the initial states $\left(\alpha_{1}, \alpha_{2}, \ldots, \alpha_{\nu}\right)$ and $\left(\alpha_{1}, \alpha_{2}+\right.$ $\left.\varepsilon, \ldots, \alpha_{\nu}\right)$, denote the resulting numbers on the vertices of $\mathcal{G}$ at time $k$ with $\mathbf{u}[k]:=\left(u_{1}[k], u_{2}[k], \ldots, u_{\nu}[k]\right)$ and $\overline{\mathbf{u}}[k]:=$ $\left(\bar{u}_{1}[k], \bar{u}_{2}[k], \ldots, \bar{u}_{\nu}[k]\right)$, respectively, for all $k \in \mathbf{N} \cup\{0\}$. Define also:

$$
g:=T_{r, \varepsilon}\left(\alpha_{1}, \alpha_{2}+\varepsilon, \ldots, \alpha_{\nu} \mid \mathcal{H}\right)
$$

For a proof by contradiction, assume that $T_{r, \varepsilon}(\boldsymbol{\alpha} \mid \mathcal{H})>g$. Observe that:

i) Since $q(\gamma)=r+1$, for all $\gamma \in(r+0.5, r+0.5+2 \varepsilon]$, it can be verified that:

$$
\begin{aligned}
& u_{1}[k]=\bar{u}_{1}[k]=\alpha_{1}, \\
& u_{i}[k], \bar{u}_{i}[k] \in(r+0.5-\varepsilon, r+0.5+\varepsilon]
\end{aligned}
$$

for all $k \in\{0,1, \ldots, g-1\}$ and $i \in \boldsymbol{\nu} \backslash\{1\}$.

ii) Using property (i) and by means of induction on $k$, one can show that if $\bar{u}_{j}[k] \leq r+0.5$ for some $j \in \boldsymbol{\nu}$ and $k \in\{0,1, \ldots, g-1\}$, then $u_{j}[k]=\bar{u}_{j}[k]$.

Let the $g^{\text {th }}$ element of $\mathcal{H}$ be the edge $(i, j)$, where $i<j$. It results from the definition of $g$ and property (i) that $i=1$ and $\bar{u}_{j}[g-1] \leq r+0.5$ (this is the only way to generate a positive action). Therefore, by properties (i) and (ii), one can write:

$$
\begin{aligned}
& u_{j}[g-1]=\bar{u}_{j}[g-1] \leq r+0.5, \\
& u_{1}[g-1]>r+0.5+\varepsilon
\end{aligned}
$$

As a result, Remark 1 leads to the conclusion that selecting the edge $(i, j)$ at time $g$ results in a positive action for the graph $\mathcal{G}(\mathcal{V}, \mathcal{E})$ with the initial state $\boldsymbol{\alpha}$; i.e. $T_{r, \varepsilon}(\boldsymbol{\alpha} \mid \mathcal{H})=g$. This contradicts the aforementioned assumption.

Proposition 2: Consider the objects $r, \mathcal{H}$ and $\zeta(\varepsilon, r, \mathcal{H})$ introduced in Proposition 1 and Definition 9. There exists a vector $\boldsymbol{\alpha}:=\left[\begin{array}{ccc}\alpha_{1} & \cdots & \alpha_{\nu}\end{array}\right]$ such that:

$$
\begin{aligned}
\zeta(\varepsilon, r, \mathcal{H}) & =T_{r, \varepsilon}(\boldsymbol{\alpha} \mid \mathcal{H}) \\
\left\{\alpha_{1}, \alpha_{2}, \ldots, \alpha_{\nu}\right\} & =\left\{r+0.5-\frac{\varepsilon}{2}, r+0.5+\frac{\varepsilon}{2}, \ldots\right. \\
& \left.r+0.5+\frac{\varepsilon}{2}, r+0.5+3 \frac{\varepsilon}{2}\right\}
\end{aligned}
$$

(note that the term $r+0.5+\frac{\varepsilon}{2}$ appears $\nu-2$ times in the above set).

Proof: It follows from Proposition 1 and Lemma 2 that there exist a vector $\boldsymbol{\alpha}=\left[\begin{array}{lll}\alpha_{1} & \cdots & \alpha_{\nu}\end{array}\right]$ and integers $\mu_{1}, \mu_{2} \in \nu$ with the properties:

- $\zeta(\varepsilon, r, \mathcal{H})=T_{r, \varepsilon}(\boldsymbol{\alpha} \mid \mathcal{H})$

- The set of inequalities:

$$
\begin{aligned}
r+0.5+\varepsilon & <\alpha_{\mu_{1}} \leq r+0.5+2 \varepsilon, \\
r+0.5-\varepsilon & <\alpha_{\mu_{2}} \leq r+0.5, \\
r+0.5 & <\alpha_{i} \leq r+0.5+\varepsilon, \quad \forall i \in \nu \backslash\left\{\mu_{1}, \mu_{2}\right\}
\end{aligned}
$$

or:

$$
\begin{aligned}
r+0.5-2 \varepsilon & <\alpha_{\mu_{1}} \leq r+0.5-\varepsilon \\
r+0.5 & <\alpha_{\mu_{2}} \leq r+0.5+\varepsilon \\
r+0.5-\varepsilon & <\alpha_{i} \leq r+0.5, \quad \forall i \in \boldsymbol{\nu} \backslash\left\{\mu_{1}, \mu_{2}\right\}
\end{aligned}
$$

holds.

Due to the symmetry, one can assume with no loss of generality that the set of inequalities given in (61) holds. It is straightforward to show (using the above properties) that $T_{r, \varepsilon}(\boldsymbol{\alpha} \mid \mathcal{H})$ is unchanged if the following replacements are made:

- $\alpha_{\mu_{1}}$ with $r+0.5+\frac{3 \varepsilon}{2}$;

- $\alpha_{\mu_{2}}$ with $r+0.5-\frac{\varepsilon}{2}$;

- $\alpha_{i}$ with $r+0.5+\frac{\varepsilon}{2}$, for any $i \in \nu \backslash\left\{\mu_{1}, \mu_{2}\right\}$.

This completes the proof.

The proof of Theorem 2 is a direct consequence of Proposition 2 given above. 